\title{
Cord blood and child plasma adiponectin levels in relation to childhood obesity risk and fat distribution up to $5 \mathrm{y}$
}

\author{
Dorothy M. Meyer', Christina Brei', Lynne Stecher', Daniela Much², Stefanie Brunner' and Hans Hauner ${ }^{1,3}$
}

BACKGROUND: Few human studies have explored the role of adiponectin in early life on growth and adipose tissue development.

METHODS: High molecular weight (HMW) and total adiponectin levels from 141 cord blood samples and plasma blood samples from 40 3-y-old children were analyzed. Associations between adiponectin levels in cord blood and child plasma, and infant/child growth and fat mass measurements up to the age of 5 y were assessed using linear regression models.

RESULTS: HMW cord blood adiponectin was positively associated with weight, BMI percentiles, and lean body mass at birth only. At 3 and 4 y, positive associations were found with cord blood adiponectin and sum of four skinfold thickness measures and percentage of body fat following adjustment for maternal and child covariates, but did not persist at $5 \mathrm{y}$. There was no significant evidence of an association between child plasma HMW adiponectin and growth or body composition characteristics at 3-5 y.

CONCLUSION: Our results do not support the hypothesis that HMW cord blood adiponectin is a useful biomarker for the prediction of adiposity at the age of $5 \mathrm{y}$. Additionally, there is no evidence that plasma HMW adiponectin levels predict body fat distribution between 3-5 y.

$c$ hildhood obesity has been steadily increasing for decades worldwide (1), which follows the trend of the general population. However, in recent years, there has been a marked rise in the number of preschool children suffering from overweight and obesity, creating an urgent need for early intervention strategies. Interventions aimed at children with obesity have shown mixed results, and rarely result in long term weight reduction (2). Recent research has demonstrated that the origin of obesity may develop in utero as a result of physiological adaptations by the fetus to intrauterine factors (3). Due to these findings, research efforts are being directed toward identifying novel biomarkers that can assist in the early prediction of childhood adiposity, with the goal of identifying children who are particularly at risk and directing preventative strategies from the onset.

Adiponectin, a hormone secreted by white adipose tissue, has been put forward as a biomarker candidate, as it is known to play a critical role in energy regulation and metabolic homeostasis (4). Obesity is characterized by a downregulation of adiponectin in adults and children from school-age through adolescence (57). Research has consistently demonstrated a strong association between decreased adiponectin levels and increased risk of type 2 diabetes (8). Moreover, low adiponectin levels in prepubescent and adolescent children are a risk factor for the development of metabolic syndrome $(9,10)$. However, the role of adiponectin in obesity and metabolic dysregulation in preschool-aged children is not as clear. To date, most studies with very young children have focused on the association between cord blood total adiponectin and birth weight and growth parameters. The only study to examine this relationship past the early postnatal period was conducted by Mantzoros et al. (11), which found a positive correlation of cord blood total adiponectin on abdominal adiposity at $3 \mathrm{y}$, assessed as the ratio of subscapular/triceps skinfolds. Other studies have investigated the association of plasma adiponectin with growth parameters (12) and fat distribution in newborns up to $2 \mathrm{y}$ of life $(13,14)$. Moreover, research has suggested that high molecular weight (HMW) adiponectin concentration, rather than total circulating adiponectin, may be a more valuable clinical bio-marker for the prediction of obesity and obesity related diseases in older children and adolescents (15). However, to our knowledge, there is no literature which considers these relationships in the preschool years.

The purpose of our study was twofold. First, this study investigated whether cord blood total and HMW adiponectin levels could predict measures of adiposity from birth to $5 \mathrm{y}$ of age. Furthermore, we examined the association of child plasma total and HMW adiponectin (at $3 \mathrm{y}$ ) with anthropometric measurements and body fat distribution in children at 3, 4, and 5 y old.

\footnotetext{
The first two authors contributed equally to this work.

${ }^{1}$ From the Else Kröner-Fresenius-Center for Nutritional Medicine, Klinikum rechts der Isar, Technische Universität München, Munich, Germany; ${ }^{2}$ Institute of Diabetes Research, Helmholtz Zentrum München, and Forschergruppe Diabetes, Klinikum rechts der Isar, Technische Universität München, Munich, Germany; ${ }^{3}$ ZIEL - Institute for Food and Health, Nutritional Medicine Unit, Technische Universität München, Freising, Germany. Correspondence: H Hauner (hans.hauner@tum.de)

Received 28 June 2016; accepted 30 October 2016; advance online publication 15 February 2017. doi:10.1038/pr.2016.275
} 


\section{METHODS}

\section{Subjects}

Data for this study were taken from the INFAT study (impact of nutritional fatty acids during pregnancy and lactation on the early human adipose tissue development) (16-19). The original study was a randomized controlled clinical trial which investigated the effect of reducing the ratio of n-6/n-3 fatty acids during pregnancy and lactation on offspring's body composition up to $1 \mathrm{y}$ of life, with an additional follow-up until the age of 5 .

The study population, design, and outcomes have been previously described $(16,17)$. Briefly, 208 healthy pregnant women with a prepregnancy BMI between 18 and $30 \mathrm{~kg} / \mathrm{m}^{2}$ were recruited before the $15^{\text {th }}$ week of gestation and randomly assigned to either a control or intervention group. The intervention group received a daily fish oil supplement consisting of $1,020 \mathrm{mg}$ DHA $+180 \mathrm{mg}$ EPA as well as dietary consultations aimed at achieving an arachidonic acid balanced diet from the $15^{\text {th }}$ week of gestation until 4 mo postpartum. The control group was provided general dietary counseling for pregnancy and lactation as per current German recommendations.

For the initial study, infant weight, height, and skinfold thickness (SFT) measurements were measured and recorded at birth, $6 \mathrm{wk}, 4$ $\mathrm{mo}$, and $1 \mathrm{y}$ of life. Abdominal ultrasound was investigated on infants at $6 \mathrm{wk}, 4 \mathrm{mo}$, and $1 \mathrm{y}$. The follow-up study assessments were then performed annually from $2-5$ y of life. For both the initial study and follow-up visits, the primary outcome was SFT measurements. In addition, abdominal magnetic resonance imaging (MRI) was performed in a subgroup of children at $5 \mathrm{y}$ of age (Department of Radiology, Klinikum rechts der Isar, Munich, Germany). Cord blood samples were collected at birth, as well as venous blood samples from children at $3 \mathrm{y}$.

Approval for the study protocol was obtained from the ethical committee of the Technische Universität München, Germany $(1479 / 06 / 2009 / 10 / 26)$. For the initial study with mother/infant pairs, written informed consent was obtained from the mother only. Written informed consent from both parents was received for the follow-up study with children aged 2-5 y and separately for the MRI measurement (from the accompanying parent).

\section{Collection of Bio-samples and Laboratory Analyses}

In total, 141 cord blood samples from the umbilical vein were collected in EDTA tubes after delivery. Venous blood, which was available from a subgroup of 403 -y-old children, was collected in EDTA tubes during the follow-up visit. Blood samples were centrifuged at $2,000 \mathrm{~g}$ at $4^{\circ} \mathrm{C}$ for $10 \mathrm{~min}$. The plasma was then aliquoted and stored at $-80^{\circ} \mathrm{C}$ until analysis.

Adiponectin concentrations in cord blood and child plasma at $3 \mathrm{y}$ were measured by a commercially available enzyme-linked immunosorbent assay (ELISA) (ALPCO Diagnostics, Salem NH) using an antibody sandwich method. To quantify HMW adiponectin, plasma was treated with a protease to digest low- and medium molecular weight adiponectin prior to performing the sandwich ELISA protocol. The ratio of HMW-to-total adiponectin was calculated for cord blood (cord blood HMW adiponectin/cord blood total adiponectin) and child plasma at 3 y (3 y HMW adiponectin/3 y total adiponectin).

\section{Anthropometric Measurements}

The infants and children were weighed and measured using standardized techniques described elsewhere $(18,19)$. BMI percentiles were calculated using German pediatric growth charts (20), SFT measurements were assessed in triplicate at four body sites on the left body axis (triceps, biceps, subscapular, and suprailiac) with a Holtain Caliper (Holtain, Crymych, UK). The mean of the three measurements for each site was used for analysis. Skinfold regression equations were computed according to Weststrate and Deurenberg (21) to determine body fat percentage and fat mass in $\mathrm{kg}$. Lean body mass was determined by subtracting body weight from fat mass.

In order to estimate abdominal subcutaneous and preperitoneal fat, abdominal ultrasonography was performed with small adaptations according to the method of Holzhauer et al. (22) at each visit by trained research assistants as described previously (18). Additionally, MRI measurements were performed to assess abdominal subcutaneous-, visceral-, and nonadipose tissue (SAT, VAT, and NAT) volumes as described by Brei et al., 2016 (19). Significant differences in growth and body composition between the two groups was not observed at any age except for higher weight and ponderal index at birth, as a result of prolonged pregnancy (16), as well as weight and BMI at $4 \mathrm{y}$ in the unadjusted analysis only (19).

\section{Statistical Analysis}

Statistical analyses were performed with SPSS version 21 (IBM, New York, NY). The cord blood and child plasma adiponectin levels were summarized by median and interquartile ranges (IQR), with differences according to sex assessed using the Mann-Whitney $U$-test. The association between adiponectin levels in cord blood and child plasma was summarized using Spearman rank correlation. Linear regression models were fitted to examine the relationship between cord blood adiponectin, blood plasma adiponectin, and child body composition characteristics. Univariate models and models adjusted for maternal prepregnancy BMI, gestational weight gain, pregnancy duration, sex (except for BMI percentiles), ponderal index at birth $\left(\mathrm{kg} / \mathrm{m}^{3}\right)$, group, and mode of infant feeding (exclusively or partially breastfed) at 4 mo postpartum (except at birth) were fitted. A two-sided $P$-value $\leq 0.05$ was considered statistically significant and no adjustment was made for multiple comparisons.

\section{RESULTS}

\section{Adiponectin Concentrations in Cord Blood and Child Plasma}

Total and HMW adiponectin concentrations in cord blood and in child plasma at $3 \mathrm{y}$ are presented in Table 1 . Median concentrations (IQR) of cord blood total and HMW adiponectin were $14.90 \mu \mathrm{g} / \mathrm{ml}(12.91 ; 18.43)$ and $10.10 \mu \mathrm{g} / \mathrm{ml}(8.25$; 12.78), respectively. In contrast, concentrations of total and HMW adiponectin in child plasma at $3 \mathrm{y}$ were $9.17 \mu \mathrm{g} / \mathrm{ml}$ $(7.82 ; 12.10)$ and $5.35 \mu \mathrm{g} / \mathrm{ml}(4.04 ; 7.41)$, respectively. No sex differences were observed in either cord blood total or HMW adiponectin. Higher cord blood adiponectin levels were significantly associated with increased child plasma adiponectin

Table 1. Adiponectin concentrations and ratios of total and HMW adiponectin in cord blood and child plasma at 3 y of age

\begin{tabular}{|c|c|c|c|c|c|c|c|}
\hline \multirow[b]{2}{*}{ Biological sample } & \multicolumn{2}{|r|}{ Total } & \multicolumn{2}{|r|}{ Males } & \multicolumn{2}{|r|}{ Females } & \multirow[b]{2}{*}{$P^{a}$} \\
\hline & $n$ & Median (IQR) & $n$ & Median (IQR) & $n$ & Median (IQR) & \\
\hline Cord blood total adiponectin, $\mu \mathrm{g} / \mathrm{ml}$ & 141 & $14.90(12.91 ; 18.43)$ & 76 & $15.05(13.23 ; 18.06)$ & 65 & $14.61(12.55 ; 19.30)$ & 0.913 \\
\hline Cord blood HMW adiponectin, $\mu \mathrm{g} / \mathrm{ml}$ & 141 & $10.10(8.25 ; 12.78)$ & 76 & $10.38(8.81 ; 12.35)$ & 65 & $9.78(7.90 ; 13.14)$ & 0.756 \\
\hline Cord blood ratio total/HMW adiponectin & 141 & $1.51(1.42 ; 1.59)$ & 76 & $1.50(1.40 ; 1.60)$ & 65 & $1.52(1.46 ; 1.58)$ & 0.130 \\
\hline Plasma child total adiponectin, $\mu \mathrm{g} / \mathrm{ml}^{\mathrm{b}}$ & 40 & $9.17(7.82 ; 12.10)$ & 20 & $8.80(7.60 ; 11.73)$ & 20 & $10.37(8.13 ; 12.33)$ & 0.317 \\
\hline Plasma child HMW adiponectin, $\mu \mathrm{g} / \mathrm{ml}^{\mathrm{b}}$ & 40 & $5.35(4.04 ; 7.41)$ & 20 & $4.60(3.68 ; 6.96)$ & 20 & $5.80(4.51 ; 8.58)$ & 0.330 \\
\hline Plasma child ratio total/HMW adiponectin & 40 & $1.74(1.63 ; 1.94)$ & 20 & $1.79(1.63 ; 1.96)$ & 20 & $1.74(1.61 ; 1.83)$ & 0.490 \\
\hline
\end{tabular}

HMW, high molecular weight; IQR, interquartile range. ${ }^{\text {DD }}$ ifferences according to sex assessed by Mann-Whitney $U$-test. ${ }^{b} M e a s u r e d$ at 3 y of age. 
(total adiponectin $0.578(P<0.001)$ (Figure 1a) and HMW adiponectin $0.558(P<0.001)$ (Figure $1 b)$ ).

\section{Cord Blood Adiponectin Levels in Relation to Child Weight and Body Composition from Birth to $5 \mathrm{y}$}

A positive relationship between HMW cord blood adiponectin and weight, BMI percentiles, fat mass, and lean body mass at birth was observed in the univariate models. In the models adjusted for maternal and child covariates, the results were consistent except for fat mass, which was no longer statistically significant (Table 2). Similar results were found for total adiponectin, and these are presented as Supplementary Table S1 online.

At later time points, no positive association was found in the unadjusted model between HMW adiponectin and child outcomes except for preperitoneal fat at $2 \mathrm{y}$, as well as sum of SFT and body fat percentage at $3 \mathrm{y}$. The adjusted model showed significant relationships with preperitoneal fat at $2 \mathrm{y}$, as well as sum of SFT, body fat percentage and fat mass at 3 y. At $4 \mathrm{y}$, relationships with sum of SFT and body fat percentage persisted, while no significant relationships were apparent in the fifth year of life. Additionally, we found no association between cord blood adiponectin and abdominal visceral and subcutaneous fat assessed by MRI at $5 \mathrm{y}$.

\section{Child Plasma Adiponectin Levels at 3 y in Relation to Child Weight and Body Composition at 3, 4, and 5 y of Life}

In contrast to cord blood, we did not find significant evidence of an association between child plasma HMW adiponectin and growth or body composition characteristics at 3-5 y in the unadjusted or adjusted model, including fat mass measured by abdominal MRI in a subgroup of children at $5 \mathrm{y}$ (Table 3). Analogous analyses for total adiponectin are given as Supplementary Table S2 online with consistent findings except for significant evidence of an association between total adiponectin concentration and lean body mass in the adjusted analysis at $3 \mathrm{y}$.

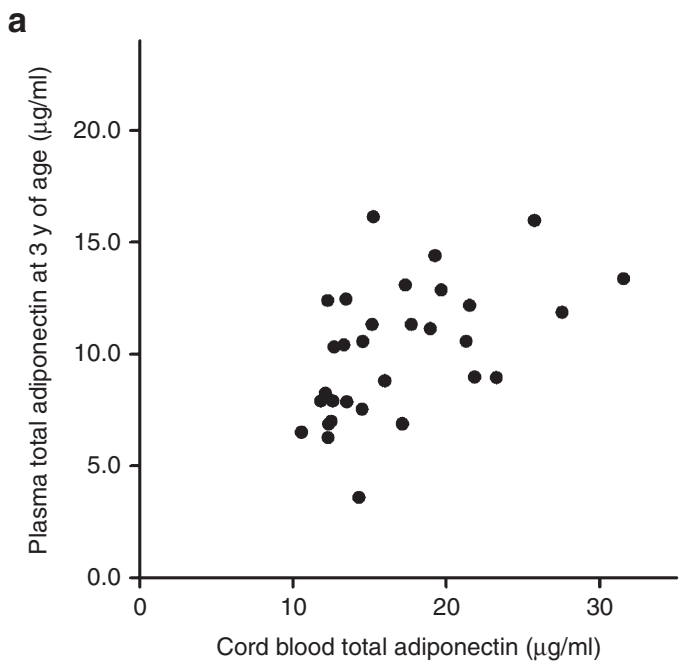

\section{DISCUSSION}

Using longitudinal data from the INFAT maternal/child cohort, this study examined the relationships of cord blood and child plasma adiponectin levels with indices of fat mass and distribution in preschool children. We found that cord blood adiponectin concentrations were higher than child plasma adiponectin levels at $3 \mathrm{y}$ of age, and that elevated cord blood adiponectin correlated with higher birth weight. The main finding of our study was that cord blood total and HMW adiponectin concentrations were associated with adiposity related parameters at 3 and $4 \mathrm{y}$ of age, assessed by SFTs and body fat percentage, but this trend did not persist to the fifth year of life. Moreover, we did not find evidence that plasma total/HMW adiponectin levels measured at $3 \mathrm{y}$ predicted adiposity at 3,4 , and $5 \mathrm{y}$.

To our knowledge, this is the first study which examines the longitudinal relationship between cord blood adiponectin concentrations and obesity risk from birth to the age of school entry with a variety of methods to assess body composition and fat distribution. While the association with cord blood adiponectin with infant growth and body composition characteristics has been examined in several previous studies, findings have been inconclusive. Whereas some studies have shown a positive association at birth and early postpartum $(11,23,24)$, others have suggested that no relationship exists $(25,26)$. Our data are consistent with previously reported findings from Mantzoros et al. (11), which found a positive correlation between cord blood total adiponectin concentration and measurements of central adiposity at $3 \mathrm{y}$. In light of recent interest in identifying adiponectin as a potential diagnostic biomarker for early identification of obesity and insulin insensitivity in children $(9,27,28)$, our study is unique in that it is the first to assess the relationship of cord blood adiponectin with body fat measurements past the age of $3 \mathrm{y}$. Moreover, as recent studies posit that HMW adiponectin is a better predictor of insulin sensitivity and obesity mediated metabolic parameters in children $(15,29)$, our findings provide greater insight into

\section{b}

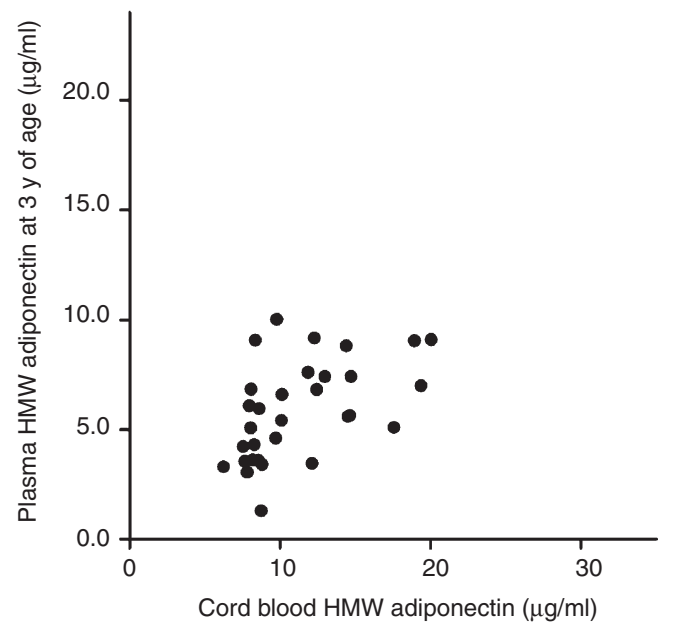

Figure 1. Association between adiponectin in cord blood and child plasma. Association of (a) cord blood total adiponectin with plasma total adiponectin at 3 y of age $(n=31)$ and (b) cord blood high molecular weight (HMW) adiponectin with plasma HMW adiponectin at 3 y of age ( $n=31)$ (a: Spearman $r_{s}=0.5778 ; P<0.001 ; \mathbf{b}:$ Spearman $\left.r_{s}=0.5581, P=0.001\right)$. 
Table 2. Cord blood HMW adiponectin in relation to infant clinical outcomes from birth to 5 y of age

\begin{tabular}{|c|c|c|c|c|c|c|}
\hline \multirow[b]{2}{*}{ Body composition variables } & \multicolumn{3}{|c|}{ Unadjusted analysis } & \multicolumn{3}{|c|}{ Adjusted analysis ${ }^{a}$} \\
\hline & $n$ & Beta $(95 \% \mathrm{Cl})$ & $P$ & $n$ & Beta $(95 \% \mathrm{Cl})$ & $P$ \\
\hline \multicolumn{7}{|l|}{ Birth } \\
\hline Weight, kg & 141 & $0.03(0.01 ; 0.05)$ & 0.004 & 139 & $0.02(0.00 ; 0.04)$ & 0.022 \\
\hline BMI percentiles & 141 & $1.20(0.05 ; 2.35)$ & 0.041 & 139 & $0.53(0.00 ; 1.05)$ & 0.050 \\
\hline Sum 4 SFTs, mm & 134 & $0.10(-0.01 ; 0.20)$ & 0.082 & 132 & $0.07(-0.05 ; 0.18)$ & 0.241 \\
\hline Fat mass, kg & 134 & $0.01(0.00 ; 0.01)$ & 0.011 & 132 & $0.01(-0.00 ; 0.01)$ & 0.070 \\
\hline Body fat (\%) & 134 & $0.09(-0.02 ; 0.20)$ & 0.113 & 132 & $0.06(-0.06 ; 0.07)$ & 0.324 \\
\hline Lean body mass, kg & 134 & $0.02(0.01 ; 0.04)$ & 0.002 & 132 & $0.02(0.00 ; 0.03)$ & 0.011 \\
\hline \multicolumn{7}{|l|}{$1 \mathrm{y}$} \\
\hline Weight, kg & 131 & $0.02(-0.03 ; 0.06)$ & 0.415 & 131 & $0.03(-0.01 ; 0.08)$ & 0.149 \\
\hline BMI percentiles & 131 & $0.72(-0.59 ; 2.03)$ & 0.281 & 131 & $0.80(-0.50 ; 2.10)$ & 0.227 \\
\hline Sum 4 SFTs, mm & 126 & $-0.03(-0.21 ; 0.16)$ & 0.770 & 126 & $0.03(-0.16 ; 0.23)$ & 0.756 \\
\hline Fat mass, kg & 126 & $0.00(-0.02 ; 0.02)$ & 0.783 & 126 & $0.01(-0.01 ; 0.03)$ & 0.373 \\
\hline Body fat (\%) & 126 & $-0.01(-0.14 ; 0.12)$ & 0.865 & 126 & $0.02(-0.11 ; 0.16)$ & 0.741 \\
\hline Lean body mass, kg & 126 & $0.02(-0.01 ; 0.05)$ & 0.283 & 126 & $0.03(-0.01 ; 0.06)$ & 0.100 \\
\hline Area sag pp, mm² & 120 & $-0.06(-0.32 ; 0.20)$ & 0.661 & 120 & $-0.06(-0.34 ; 0.22)$ & 0.657 \\
\hline Area sag sc, mm² & 121 & $0.39(-0.22 ; 0.99)$ & 0.208 & 121 & $0.42(-0.21 ; 1.06)$ & 0.190 \\
\hline Area ax sc, mm² & 123 & $0.29(-0.41 ; 0.99)$ & 0.415 & 123 & $0.24(-0.49 ; 0.97)$ & 0.517 \\
\hline \multicolumn{7}{|l|}{$2 y$} \\
\hline Weight, kg & 130 & $0.04(-0.02 ; 0.10)$ & 0.240 & 130 & $0.04(-0.02 ; 0.10)$ & 0.163 \\
\hline BMI percentiles & 130 & $0.96(-0.27 ; 2.18)$ & 0.124 & 130 & $0.98(-0.27 ; 2.23)$ & 0.122 \\
\hline Sum 4 SFTs, mm & 90 & $0.18(-0.02 ; 0.39)$ & 0.082 & 90 & $0.21(-0.01 ; 0.43)$ & 0.056 \\
\hline Fat mass, kg & 90 & $0.02(-0.01 ; 0.06)$ & 0.114 & 90 & $0.03(-0.01 ; 0.06)$ & 0.126 \\
\hline Body fat (\%) & 90 & $0.12(-0.02 ; 0.26)$ & 0.102 & 90 & $0.14(-0.02 ; 0.29)$ & 0.079 \\
\hline Lean body mass, kg & 90 & $0.03(-0.04 ; 0.09)$ & 0.400 & 90 & $0.02(-0.05 ; 0.08)$ & 0.542 \\
\hline Area sag pp, mm² & 89 & $0.60(0.12 ; 1.07)$ & 0.014 & 89 & $0.61(0.09 ; 1.12)$ & 0.022 \\
\hline Area sag sc, mm² & 89 & $0.47(-0.21 ; 1.15)$ & 0.173 & 89 & $0.71(-0.02 ; 1.44)$ & 0.056 \\
\hline Area ax sc, $\mathrm{mm}^{2}$ & 89 & $0.40(-0.38 ; 1.19)$ & 0.307 & 89 & $0.66(-0.16 ; 1.48)$ & 0.111 \\
\hline \multicolumn{7}{|l|}{$3 y$} \\
\hline Weight, kg & 124 & $0.02(-0.06 ; 0.10)$ & 0.610 & 124 & $0.03(-0.05 ; 0.11)$ & 0.402 \\
\hline BMI percentiles & 124 & $0.35(-0.84 ; 1.83)$ & 0.561 & 124 & $0.62(-0.56 ; 1.80)$ & 0.300 \\
\hline Sum 4 SFTs, mm & 90 & $0.23(0.02 ; 0.45)$ & 0.030 & 90 & $0.30(0.10 ; 0.51)$ & 0.005 \\
\hline Fat mass, kg & 90 & $0.03(-0.00 ; 0.07)$ & 0.085 & 90 & $0.04(0.00 ; 0.08)$ & 0.041 \\
\hline Body fat (\%) & 90 & $0.16(0.00 ; 0.31)$ & 0.046 & 90 & $0.21(0.06 ; 0.35)$ & 0.006 \\
\hline Lean body mass, kg & 99 & $0.01(-0.06 ; 0.09)$ & 0.719 & 90 & $0.01(-0.07 ; 0.08)$ & 0.894 \\
\hline Area sag pp, mm² & 80 & $0.57(-0.14 ; 1.28)$ & 0.114 & 80 & $0.55(-0.22 ; 1.33)$ & 0.161 \\
\hline Area sag sc, mm² & 80 & $0.56(-0.19 ; 1.30)$ & 0.142 & 80 & $0.73(-0.05 ; 1.51)$ & 0.066 \\
\hline Area ax sc, mm² & 79 & $0.63(-0.49 ; 1.74)$ & 0.266 & 79 & $0.87(-0.29 ; 2.04)$ & 0.139 \\
\hline \multicolumn{7}{|l|}{$4 y$} \\
\hline Weight, kg & 124 & $0.03(-0.06 ; 0.11)$ & 0.553 & 124 & $0.04(-0.05 ; 0.13)$ & 0.411 \\
\hline BMI percentiles & 124 & $0.57(-0.63 ; 1.77)$ & 0.351 & 124 & $0.73(-0.48 ; 1.93)$ & 0.236 \\
\hline Sum 4 SFTs, mm & 80 & $0.18(-0.04 ; 0.40)$ & 0.111 & 80 & $0.24(0.00 ; 0.47)$ & 0.048 \\
\hline Fat mass, kg & 80 & $0.03(-0.01 ; 0.07)$ & 0.165 & 80 & $0.04(-0.01 ; 0.08)$ & 0.124 \\
\hline Body fat (\%) & 80 & $0.13(-0.04 ; 0.30)$ & 0.126 & 80 & $0.19(0.02 ; 0.35)$ & 0.027 \\
\hline Lean body mass, kg & 80 & $0.03(-0.08 ; 0.13)$ & 0.609 & 80 & $0.00(-0.11 ; 0.11)$ & 0.962 \\
\hline Area sag $p p, \mathrm{~mm}^{2}$ & 75 & $0.08(-0.80 ; 0.96)$ & 0.854 & 75 & $0.06(-0.91 ; 1.04)$ & 0.896 \\
\hline Area sag sc, mm² & 74 & $0.41(-0.36 ; 1.18)$ & 0.292 & 74 & $0.61(-0.21 ; 1.43)$ & 0.143 \\
\hline Area ax sc, mm² & 75 & $0.33(-0.78 ; 1.45)$ & 0.553 & 75 & $0.62(-0.60 ; 1.84)$ & 0.315 \\
\hline \multicolumn{7}{|l|}{$5 y$} \\
\hline Weight, kg & 120 & $-0.00(-0.13 ; 0.13)$ & 0.986 & 120 & $0.02(-0.11 ; 0.15)$ & 0.752 \\
\hline BMI percentiles & 119 & $0.12(-1.17 ; 1.42)$ & 0.851 & 119 & $0.49(-0.82 ; 1.79)$ & 0.460 \\
\hline Sum 4 SFTs, mm & 89 & $0.06(-0.21 ; 0.34)$ & 0.646 & 89 & $0.12(-0.17 ; 0.40)$ & 0.408 \\
\hline Fat mass, kg & 89 & $0.01(-0.05 ; 0.07)$ & 0.710 & 89 & $0.02(-0.04 ; 0.08)$ & 0.551 \\
\hline Body fat (\%) & 89 & $0.06(-0.14 ; 0.26)$ & 0.545 & 89 & $0.08(-0.10 ; 0.27)$ & 0.363 \\
\hline Lean body mass, kg & 89 & $-0.01(-0.12 ; 0.09)$ & 0.848 & 89 & $0.00(-0.11 ; 0.10)$ & 0.962 \\
\hline Area sag pp, mm² & 74 & $0.32(-0.51 ; 1.15)$ & 0.446 & 74 & $0.56(-0.33 ; 1.46)$ & 0.214 \\
\hline Area sag sc, mm² & 75 & $0.17(-0.58 ; 0.92)$ & 0.658 & 75 & $0.26(-0.57 ; 1.10)$ & 0.534 \\
\hline Area ax sc, mm² & 76 & $-0.00(-1.10 ; 1.09)$ & 0.996 & 76 & $0.15(-1.07 ; 1.38)$ & 0.804 \\
\hline SAT volume, $\mathrm{cm}^{3}$ & 33 & $0.33(-15.10 ; 15.76)$ & 0.965 & 33 & $7.22(-10.17 ; 24.62)$ & 0.400 \\
\hline VAT volume, $\mathrm{cm}^{3}$ & 33 & $-0.10(-3.40 ; 3.21)$ & 0.954 & 33 & $1.57(-2.20 ; 5.34)$ & 0.399 \\
\hline NAT volume, $\mathrm{cm}^{3}$ & 33 & $-4.75(-37.90 ; 28.39)$ & 0.772 & 33 & $4.00(-32.40 ; 40.40)$ & 0.823 \\
\hline
\end{tabular}


Table 3. HMW adiponectin in plasma of 3 -y-old children in relation to their body composition at 3, 4, and $5 \mathrm{y}$ of age

\begin{tabular}{|c|c|c|c|c|c|}
\hline \multirow[b]{2}{*}{ Body composition variables } & \multirow[b]{2}{*}{$n$} & \multicolumn{2}{|c|}{ Unadjusted analysis } & \multicolumn{2}{|c|}{ Adjusted analysis ${ }^{a}$} \\
\hline & & Beta $(95 \% \mathrm{Cl})$ & $P$ & Beta $(95 \% \mathrm{Cl})$ & $P$ \\
\hline \multicolumn{6}{|l|}{$3 y$} \\
\hline Weight, kg & 40 & $0.13(-0.10 ; 0.35)$ & 0.258 & $0.18(-0.06 ; 0.42)$ & 0.143 \\
\hline BMI percentiles & 40 & $-0.90(-4.70 ; 2.91)$ & 0.636 & $-1.05(-5.10 ; 3.01)$ & 0.603 \\
\hline Sum 4 SFTs, mm & 39 & $-0.06(-0.47 ; 0.58)$ & 0.822 & $-0.01(-0.61 ; 0.59)$ & 0.970 \\
\hline Fat mass, kg & 39 & $0.03(-0.05 ; 0.11)$ & 0.434 & $0.03(-0.06 ; 0.12)$ & 0.548 \\
\hline Body fat (\%) & 39 & $0.04(-0.34 ; 0.41)$ & 0.852 & $-0.03(-0.44 ; 0.38)$ & 0.881 \\
\hline Lean body mass, kg & 39 & $0.10(-0.06 ; 0.26)$ & 0.226 & $0.14(-0.04 ; 0.31)$ & 0.113 \\
\hline Area sag $\mathrm{pp}, \mathrm{mm}^{2}$ & 39 & $-0.06(-1.56 ; 1.43)$ & 0.932 & $-0.18(-1.65 ; 1.28)$ & 0.802 \\
\hline Area sag $\mathrm{sc}, \mathrm{mm}^{2}$ & 39 & $0.07(-1.60 ; 1.74)$ & 0.931 & $-0.21(-2.09 ; 1.67)$ & 0.821 \\
\hline Area ax sc, $\mathrm{mm}^{2}$ & 39 & $-0.22(-2.75 ; 2.31)$ & 0.860 & $-0.44(-3.20 ; 2.30)$ & 0.742 \\
\hline \multicolumn{6}{|l|}{$4 y$} \\
\hline Weight, kg & 38 & $0.15(-0.11 ; 0.42)$ & 0.247 & $0.19(-0.11 ; 0.49)$ & 0.204 \\
\hline BMI percentiles & 38 & $0.20(-3.94 ; 4.34)$ & 0.922 & $0.32(-4.22 ; 4.87)$ & 0.886 \\
\hline Sum 4 SFTs, mm & 34 & $0.09(-0.46 ; 0.64)$ & 0.745 & $0.09(-0.61 ; 0.78)$ & 0.798 \\
\hline Fat mass, $\mathrm{kg}$ & 34 & $0.04(-0.06 ; 0.14)$ & 0.382 & $0.04(-0.08 ; 0.16)$ & 0.513 \\
\hline Body fat (\%) & 34 & $0.11(-0.29 ; 0.51)$ & 0.591 & $0.09(-0.41 ; 0.58)$ & 0.725 \\
\hline Lean body mass, kg & 34 & $0.10(-0.12 ; 0.31)$ & 0.367 & $0.11(-0.13 ; 0.36)$ & 0.348 \\
\hline Area sag $\mathrm{pp}, \mathrm{mm}^{2}$ & 34 & $-1.21(-3.19 ; 0.77)$ & 0.222 & $-1.50(-3.86 ; 0.85)$ & 0.200 \\
\hline Area sag $\mathrm{sc}, \mathrm{mm}^{2}$ & 34 & $0.06(-1.63 ; 1.75)$ & 0.943 & $-0.11(-2.17 ; 1.95)$ & 0.915 \\
\hline Area ax sc, $\mathrm{mm}^{2}$ & 35 & $0.24(-1.95 ; 2.43)$ & 0.825 & $0.77(-1.88 ; 3.42)$ & 0.554 \\
\hline \multicolumn{6}{|l|}{$5 y$} \\
\hline Weight, kg & 37 & $0.13(-0.23 ; 0.49)$ & 0.467 & $0.18(-0.26 ; 0.62)$ & 0.414 \\
\hline BMI percentiles & 37 & $-0.85(-4.95 ; 3.26)$ & 0.678 & $-0.62(-5.50 ; 4.27)$ & 0.798 \\
\hline Sum 4 SFTs, mm & 37 & $0.09(-0.68 ; 0.87)$ & 0.807 & $0.08(-0.85 ; 1.00)$ & 0.870 \\
\hline Fat mass, $\mathrm{kg}$ & 37 & $0.05(-0.11 ; 0.20)$ & 0.560 & $0.05(-0.14 ; 0.23)$ & 0.615 \\
\hline Body fat (\%) & 37 & $0.10(-0.46 ; 0.66)$ & 0.718 & $0.06(-0.55 ; 0.66)$ & 0.847 \\
\hline Lean body mass, kg & 37 & $0.09(-0.17 ; 0.34)$ & 0.508 & $0.13(-0.16 ; 0.42)$ & 0.361 \\
\hline Area sag $\mathrm{pp}, \mathrm{mm}^{2}$ & 34 & $-0.28(-2.03 ; 1.47)$ & 0.747 & $0.08(-2.01 ; 2.17)$ & 0.936 \\
\hline Area sag sc, $\mathrm{mm}^{2}$ & 34 & $0.55(-1.31 ; 2.41)$ & 0.551 & $-0.03(-2.26 ; 2.21)$ & 0.981 \\
\hline Area ax sc, $\mathrm{mm}^{2}$ & 35 & $-0.10(-2.87 ; 2.67)$ & 0.943 & $-0.64(-3.98 ; 2.71)$ & 0.699 \\
\hline SAT volume, $\mathrm{cm}^{3}$ & 22 & $-11.44(-42.95 ; 20.06)$ & 0.457 & $-17.19(-57.76 ; 23.38)$ & 0.377 \\
\hline VAT volume, $\mathrm{cm}^{3}$ & 22 & $-0.98(-7.51 ; 5.55)$ & 0.758 & $-0.60(-9.02 ; 7.82)$ & 0.880 \\
\hline NAT volume, $\mathrm{cm}^{3}$ & 22 & $11.19(-54.53 ; 76.90)$ & 0.726 & $-18.98(-83.87 ; 45.91)$ & 0.538 \\
\hline
\end{tabular}

Data are presented as the regression coefficient beta $(95 \% \mathrm{Cl})$ from linear regression analyses. ${ }^{2}$ Adjusted for maternal prepregnancy BMI, gestational weight gain, pregnancy duration, sex (except for BMl percentiles), ponderal index at birth, group, and mode of infant feeding (exclusively or partially breastfed) at 4 mo postpartum (except at birth). $P$-values $\leq 0.05$ are given in bold. Area ax sc, area of subcutaneous fat in axial plane; area sag pp, area of preperitoneal fat in sagittal plane; area sag sc, area of subcutaneous fat in sagittal plane; BMI, body mass index; HMW, high molecular weight; NAT, nonadipose tissue; SAT, subcutaneous adipose tissue; SFT, skinfold thickness; VAT, visceral adipose tissue.

the relationship of the metabolically active isoform of adiponectin with clinical outcomes in young children. Our results showed similar associations between total and HMW adiponectin with indices of adiposity in the study cohort. Hence, our results cannot confirm the specific role that HMW adiponectin plays in relation to fat distribution in a young pediatric population.

The mean values of child plasma total adiponectin concentration in our cohort are in agreement with reference ranges for European children published by the IDEFICS study (30). We observed no association with child plasma total and HMW adiponectin concentrations and adiposity. Consistent with our results, a study with 1 and 2-y-old children demonstrated no relationship with total adiponectin and weight or BMI (14). In contrast to preschool children, several studies have documented an inverse relationship between circulating adiponectin and measurements of body fat that manifests around the age of $6-8$ y $(6,9,31)$. A recent Europe wide study of 459 children 
confirms these findings, showing no relationship between adiponectin and growth parameters at $5.5 \mathrm{y}$, but demonstrating a negative correlation with BMI z-scores at 8 y (32).

Throughout the course of a child's development, adiponectin expression follows a markedly different pattern from that of adulthood, not only in total levels, but also in relationship to growth parameters and fat distribution. While adiponectin is exclusively secreted from adipocytes in adults, expression of the hormone has been observed in the second and third trimesters in fetal skeletal muscle, small intestine, and connective tissue (33). Adiponectin improves insulin sensitivity, and as insulin is a key growth promoter during fetal development, this underscores the pivotal role that adiponectin plays in fetal tissue growth and development. Newborns have adiponectin concentrations up to sevenfold greater than adults $(23,24)$, with declining levels after birth, and which continue to decrease with age $(27,34)$. Notwithstanding the general downward trend, prepubescent girls have higher adiponectin levels than boys, independent of BMI (35). Nevertheless, adiponectin levels in both boys and girls during childhood and adolescence remain notably higher from those observed in adulthood $(24,32)$. Furthermore, in direct contrast to observations in the adult population, adiponectin in newborns has positive correlations to growth parameters and fat mass $(24,36,37)$, with a shift in this relationship occurring around school entry age to an inverse association $(6,31)$. This evidence suggests that the role which adiponectin plays on metabolic functions and growth in utero and throughout childhood is complex and may differ fundamentally from its relationship with obesity and obesity related diseases found in adults.

A particular strength of our study is our measurement of body composition and fat distribution with several methods, including subcutaneous and visceral adipose tissue measurements assessed by abdominal MRI in a subgroup of 5 -y-old children, which is considered the gold standard (38). Moreover, our study is unique in that we have analyzed not only cord blood, but also child plasma adiponectin concentration, in both of its monomeric and multimeric HMW isoforms. These data provide us with a deeper understanding of the relationship of adiponectin on the distribution of body fat in early childhood. Additionally, our study is a longitudinal design, and includes repeated anthropometric measurements, allowing us to observe changes in body composition over a period of $5 \mathrm{y}$. However, given the relatively small study population, our results should be interpreted with caution and may not be generalizable.

In conclusion, our data do not provide evidence that cord blood adiponectin is a useful biomarker to predict adiposity at the age of school entry, although we found some associations with indices of obesity earlier in childhood. Based on our results, we additionally cannot conclude that plasma total or HMW adiponectin levels measured at 3 y predict body fat distribution at the age of $3-5 \mathrm{y}$.

\section{SUPPLEMENTARY MATERIAL}

Supplementary material is linked to the online version of the paper at http://www.nature.com/pr

\section{ACKNOWLEDGMENTS}

We are grateful to all parents with their children for participating in the INFAT study. We thank Manuela Hubersberger for laboratory analyses.

\section{STATEMENT OF FINANCIAL SUPPORT}

Supported by grants from the Else Kröner-Fresenius Foundation, Bad Homburg; the International Unilever Foundation, Hamburg; the European Union-funded Early Nutrition Programming Project (EARNEST) consortium (FOOD-CT-2005-007036); the German Ministry of Education and Research via the Competence Network Obesity (Kompetenznetz Adipositas, 01GI0842); and Danone Research-Centre for Specialised Nutrition, Friedrichsdorf, Germany. There was no intervention from any sponsor with any of the research aspects of the study including study design, intervention, data collection, analysis, and interpretation, or writing of the manuscript.

Trial registry: ClinicalTrials.gov, number ID NCT00362089, http://clinicaltrials.gov/ct2/show/NCT00362089.

Disclosure: The authors declare no conflict of interest.

\section{REFERENCES}

1. Wang Y, Lobstein T. Worldwide trends in childhood overweight and obesity. Int J Pediatr Obes 2006;1:11-25.

2. Periodic health examination, 1994 update: 1 . Obesity in childhood. Canadian Task Force on the periodic health examination. Cmaj 1994;150: 871-9.

3. Alfaradhi MZ, Ozanne SE. Developmental programming in response to maternal overnutrition. Front Genet 2011;2:27.

4. Rosen ED, Spiegelman BM. Adipocytes as regulators of energy balance and glucose homeostasis. Nature 2006;444:847-53.

5. Weiss R, Dufour S, Groszmann A, et al. Low adiponectin levels in adolescent obesity: a marker of increased intramyocellular lipid accumulation. J Clin Endocrinol Metab 2003;88:2014-8.

6. Asayama K, Hayashibe H, Dobashi K, et al. Decrease in serum adiponectin level due to obesity and visceral fat accumulation in children. Obes Res 2003;11:1072-9.

7. Weyer C, Funahashi T, Tanaka S, et al. Hypoadiponectinemia in obesity and type 2 diabetes: close association with insulin resistance and hyperinsulinemia. J Clin Endocrinol Metab 2001;86:1930-5.

8. Li S, Shin HJ, Ding EL, van Dam RM. Adiponectin levels and risk of type 2 diabetes: a systematic review and meta-analysis. JAMA 2009;302:179-88.

9. Ogawa Y, Kikuchi T, Nagasaki K, Hiura M, Tanaka Y, Uchiyama M. Usefulness of serum adiponectin level as a diagnostic marker of metabolic syndrome in obese Japanese children. Hypertens Res 2005;28:51-7.

10. Shafiee G, Ahadi Z, Qorbani M, et al. Association of adiponectin and metabolic syndrome in adolescents: the caspian- III study. J Diabetes Metab Disord 2015;14:89.

11. Mantzoros CS, Rifas-Shiman SL, Williams CJ, Fargnoli JL, Kelesidis T, Gillman MW. Cord blood leptin and adiponectin as predictors of adiposity in children at 3 years of age: a prospective cohort study. Pediatrics 2009;123:682-9.

12. Volberg V, Heggeseth B, Harley K, et al. Adiponectin and leptin trajectories in Mexican-American children from birth to 9 years of age. PLoS One 2013;8:e77964.

13. Bozzola E, Meazza C, Arvigo M, et al. Role of adiponectin and leptin on body development in infants during the first year of life. Ital J Pediatr 2010;36:26.

14. Iñiguez G, Soto N, Avila A, et al. Adiponectin levels in the first two years of life in a prospective cohort: relations with weight gain, leptin levels and insulin sensitivity. J Clin Endocrinol Metab 2004;89:5500-3.

15. Araki S, Dobashi K, Kubo K, Asayama K, Shirahata A. High molecular weight, rather than total, adiponectin levels better reflect metabolic abnormalities associated with childhood obesity. J Clin Endocrinol Metab 2006;91:5113-6.

16. Hauner H, Much D, Vollhardt C, et al. Effect of reducing the n-6:n-3 longchain PUFA ratio during pregnancy and lactation on infant adipose tissue growth within the first year of life: an open-label randomized controlled trial. Am J Clin Nutr 2012;95:383-94.

17. Hauner H, Vollhardt C, Schneider KT, Zimmermann A, Schuster T, Amann-Gassner $\mathrm{U}$. The impact of nutritional fatty acids during pregnancy 
and lactation on early human adipose tissue development. Rationale and design of the INFAT study. Ann Nutr Metab 2009;54:97-103.

18. Brei C, Much D, Heimberg E, et al. Sonographic assessment of abdominal fat distribution during the first year of infancy. Pediatr Res 2015;78:342-50.

19. Brei C, Stecher L, Much D, et al. Reduction of the n-6:n-3 long-chain PUFA ratio during pregnancy and lactation on offspring body composition: follow-up results from a randomized controlled trial up to $5 \mathrm{y}$ of age. Am J Clin Nutr 2016;103:1472-81.

20. Kromeyer-Hauschild K, Wabitsch M, Kunze D, et al. Percentiles of body mass index in children and adolescents evaluated from different regional German studies. Monatsschr Kinderheilkd 2001;8:807-19.

21. Weststrate JA, Deurenberg P. Body composition in children: proposal for a method for calculating body fat percentage from total body density or skinfold-thickness measurements. Am J Clin Nutr 1989;50:1104-15.

22. Holzhauer S, Zwijsen RM, Jaddoe VW, et al. Sonographic assessment of abdominal fat distribution in infancy. Eur J Epidemiol 2009;24:521-9.

23. Sivan E, Mazaki-ToviS, Pariente C, et al. Adiponectin in human cord blood: relation to fetal birth weight and gender. J Clin Endocrinol Metab 2003;88:5656-60.

24. Kotani Y, Yokota I, Kitamura S, Matsuda J, Naito E, Kuroda Y. Plasma adiponectin levels in newborns are higher than those in adults and positively correlated with birth weight. Clin Endocrinol (Oxf) 2004;61:418-23.

25. Lindsay RS, Walker JD, Havel PJ, Hamilton BA, Calder AA, Johnstone FD; Scottish Multicentre Study of Diabetes Pregnancy. Adiponectin is present in cord blood but is unrelated to birth weight. Diabetes Care 2003;26:2244-9.

26. Fonseca MJ, Santos AC. Umbilical cord blood adipokines and newborn weight change. Arch Gynecol Obstet 2015;291:1037-40.

27. Jeffery AN, Murphy MJ, Metcalf BS, et al. Adiponectin in childhood. Int J Pediatr Obes 2008;3:130-40.

28. Barkin S, Rao Y, Smith P, Po'e E. A novel approach to the study of pediatric obesity: a biomarker model. Pediatr Ann 2012;41:250-6.
29. Nishimura R, Morimoto A, Matsudaira T, et al. Ratio of high-, medium-, and low-molecular weight serum adiponectin to the total adiponectin value in children. J Pediatr 2007;151:545-7, 547.e1-2.

30. Erhardt E, Foraita R, Pigeot I, et al.; IDEFICS consortium. Reference values for leptin and adiponectin in children below the age of 10 based on the IDEFICS cohort. Int J Obes (Lond) 2014;38 Suppl 2:S32-8.

31. Stefan N, Bunt JC, Salbe AD, Funahashi T, Matsuzawa Y, Tataranni PA. Plasma adiponectin concentrations in children: relationships with obesity and insulinemia. J Clin Endocrinol Metab 2002;87:4652-6.

32. Gruszfeld D, Kułaga Z, Wierzbicka A, et al. Leptin and adiponectin serum levels from infancy to school age: factors influencing tracking. Child Obes 2016;12:179-87.

33. Corbetta S, Bulfamante G, Cortelazzi D, et al. Adiponectin expression in human fetal tissues during mid- and late gestation. J Clin Endocrinol Metab 2005;90:2397-402.

34. Murphy MJ, Hosking J, Metcalf BS, et al. Distribution of adiponectin, leptin, and metabolic correlates of insulin resistance: a longitudinal study in British children; 1: Prepuberty (EarlyBird 15). Clin Chem 2008;54: 1298-306.

35. Ong KK, Frystyk J, Flyvbjerg A, Petry CJ, Ness A, Dunger DB. Sex-discordant associations with adiponectin levels and lipid profiles in children. Diabetes 2006;55:1337-41.

36. Kamoda T, Saitoh H, Saito M, Sugiura M, Matsui A. Serum adiponectin concentrations in newborn infants in early postnatal life. Pediatr Res 2004;56:690-3.

37. Mantzoros C, Petridou E, Alexe DM, et al. Serum adiponectin concentrations in relation to maternal and perinatal characteristics in newborns. Eur J Endocrinol 2004;151:741-6.

38. Horan M, Gibney E, Molloy E, McAuliffe F. Methodologies to assess paediatric adiposity. Ir J Med Sci 2015;184:53-68. 